\title{
THE CAPACITANCE EFFECT ON THE TRANSFER OF GAS OR HEAT BETWEeN A BUBBLE AND THE CONTINUOUS PHASE IN A GAS-SOLID FLUIDIZED BED*
}

\author{
RYOZO TOEI, RYUICHI MATSUNO**, HIROYUKI HOTTA***, \\ MORIHIRO OICHI AND YUKIO FUJINE \\ Department of Chemical Engineering, Kyoto University, Kyoto, Japan
}

\begin{abstract}
The contribution of the particle flow (around and in a bubble) to the transfer mechanism of gas or heat between a bubble and the continuous phase was studied in a gassolid fluidized bed. Two effects of particles were considered, that is, 1) the capacitance effect of particles flowing through the cloud and 2) of those falling in a bubble. Simple models were proposed for each effect. The validities of models were confirmed by experiments of gas adsorption and heat transfer, and it was confirmed that the volumetric fraction of particles in the bubble was $\varepsilon_{p}=(1 \sim 2) \times 10^{-3}$.
\end{abstract}

\section{Introduction}

Gas transfer between a bubble and the continuous phase plays an important role when fluidized beds are used as reactors, dryers, adsorbers and heat exchangers, etc.. This problem has been discussed by many workers ${ }^{2,3,6,8,9,11,12)}$. The authors have already studied gas transfer using non-adsorptive particles and found that it was caused by the diffusion of gas from the cloud surface and the periodical shedding-out of gas from the cloud. In the above case, the effect of particle flow is indirect, that is, it affects only the gas flow pattern.

But when gas adsorption or catalytic reaction using adsorptive particles and heat transfer-controlling operations such as sand cracking or heat exchange are performed using fluidized beds, the particle flow around and in a bubble has the controlling effect on the transfer rate, since the adsorptive equilibrium constant $m$ or the heat capacity ratio of particle to gas $C_{p} \rho_{p} / C_{f} \rho_{f}$ is large, on the order of $10^{2} \sim 10^{3}$, and so a large amount of adsorptive gas or heat is absorbed by particles.

Two effects of particle flow on the transfer mechanism should be considered. 1) One is the effect of particles flowing through the cloud. This effect was first proposed by Miyauchi ${ }^{7)}$ as the adsorptive capacitance effect of particles. And Miyauchi and Moro$\mathrm{oka}^{8)}$ studied the mass transfer rate in the case without and with chemical reaction, taking this effect into

\footnotetext{
* Received on January 5, 1972

Presented at the 5th Autumn Meeting of the Soc. of Chem. Engrs., Japan, October 7, 1970

** Dept. of Food Science and Technology, Fac. of Agri., Kyoto Univ.

*** Mitsui Toatsu Chemicals Co., Ltd., Tokyo
}

account. 2) The other is the effect of particles falling in a bubble. These two effects occur simultaneously. It is the aim of this paper to make clear the roles of these two effects on the transfer mechanism between a bubble and the continuous phase.

These effects were investigated in the cases of gas adsorption and heat transfer. Simple models are suggested for each of these effects and the transfer coefficients from models are compared with those obtained from experiments of transfer of adsorptive gas (Freon 12-air) between a two-dimensional bubble and the continuous phase and of heat between a threedimensional hot air bubble and the continuous phase.

\section{Models and Theoretical Considerations}

In a gas-solid fluidized bed, the coefficient of gas interchange $k_{g t}$ or heat transfer $h_{g t}$, respectively, between a bubble and the continuous phase is defined as follows.

$$
\begin{aligned}
& -V_{b}\left(d C_{g} / d \theta\right)=k_{g t} A_{b}\left(C_{g}-C_{e}\right) \\
& -V_{b} C_{f} \rho_{f}\left(d t_{g} / d \theta\right)=h_{g t} A_{b}\left(t_{g}^{\prime}-t_{e}^{\prime}\right)=h_{g t} A_{b} t_{g}
\end{aligned}
$$

These $k_{g t}$ and $h_{g t}$ should be affected by the following four effects, namely 1) the capacitance effect of particles flowing through the cloud, 2) the diffusion of gas from the cloud surface, 3 ) the periodical shedding-out of gas from the cloud and 4) the capacitance effect of particles falling in a bubble.

The shape of the bubble is approximated by a cylinder for a two-dimensional bubble. So the volume $V_{b}$ and the surface area $A_{b}$ of the bubble are calculated as follows.

$$
\begin{aligned}
& V_{b}=(\pi / 4) D_{b}^{2} d \\
& A_{b}=\pi D_{b} d
\end{aligned}
$$

For the three-dimensional bubble, the shape is approximated by a spherical cap, as shown in Fig. $\mathbf{1}^{13}$. 


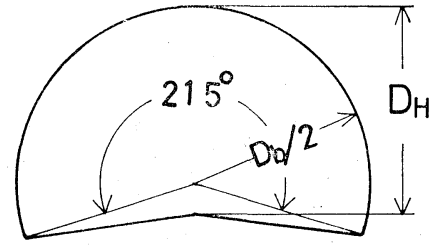

Fig. 1 The shape of three-dimensional bubble (glass beads)

$$
\begin{aligned}
V_{b} & =2.0 D_{H}^{3} \\
D_{b} & =(2 / 1.14) D_{H}
\end{aligned}
$$

And the surface area $A_{b}$ is defined as

$$
A_{b}=\pi D_{b}^{2}
$$

In the following calculations and experiments, the concentration of gas in the continuous phase $C_{e}$ is taken as zero, and the relative temperature, that is, the temperature difference from the temperature of the continuous phase, $t_{g}=\left(t_{g}^{\prime}-t_{e}^{\prime}\right)$, is used for convenience.

\subsection{The capacitance effect of particles flowing through a cloud}

A model to evaluate this effect on the gas interchange or heat transfer mechanism is suggested. The explanation of the model is performed for the case of gas adsorption.

As shown in Fig. 2, the particles flow into the cloud with flow rate $W$ and concentration $C=0$, and flow out from the cloud at the bottom of the bubble to the continuous phase after contacting with the circulating gas in the cloud in parallel. The circulating gas flows into the cloud with flow rate $G$ and concentration $C_{g}=C_{g I}$, and returns to the bubble at the bottom of the bubble with concentration $C_{\text {gout }}$. For this model the following four assumptions are made, namely 1) all amounts of the circulating gas from the bubble and the particles from the continuous phase enter from the top of the cloud, 2) the gas concentration is uniform and constant in the bubble, 3) the diffusion of gas from the cloud surface is not considered and 4) the effect of the wake is negligible. Applicability of the third assumption will be tested after calculation. Basically this model is similar to Miyauchi and Morooka's model ${ }^{8)}$ with the exception of their assumption of instantaneous adsorption equilibrium.

Gas adsorption in a particle is expressed by

$$
\frac{\partial C(\theta, r)}{\partial \theta}=\frac{\mathscr{D}_{e}}{m r^{2}} \frac{\partial}{\partial r}\left\{r^{2} \frac{\partial C(\theta, r)}{\partial r}\right\}
$$

where the adsorptive equilibrium constant $m$ is defined by the relation, $C_{s}=m C$.

Mass balance in an infinitely small distance $d x$ in the cloud is

$$
-G \frac{d C_{g}}{d x}=\frac{A\left(1-\varepsilon_{m f}\right) 4 \pi r_{p}^{2}}{(4 / 3) \pi r_{p}^{3}} k_{g}\left\{C_{g}-C\left(\theta, r_{p}\right)\right\}
$$

Using particle velocity $u_{p}$, the distance $x$ in Fig. 2 is related to time $\theta$ by

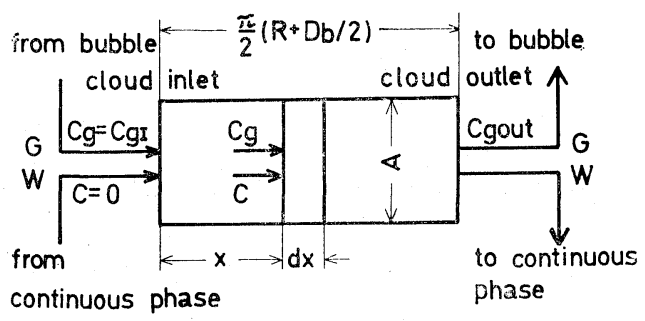

Fig. 2 The model of the flow of gas and particles in cloud

$$
A d x=A u_{p} d \theta=W d \theta /\left(1-\varepsilon_{m f}\right)
$$

$\mathrm{Eq} .(9)$ is transformed as follows.

$$
-\frac{d C_{g}}{d \theta}=\frac{3 W}{r_{p} G} k_{g}\left\{C_{g}(\theta)-C\left(\theta, r_{p}\right)\right\}
$$

The value of $k_{g}$ is calculated by the equation ${ }^{10)}$.

$$
S h=2+0.60 R e^{1 / 2} S c^{1 / 3}
$$

Boundary and initial conditions are

$$
\begin{array}{ll}
\text { at } r=0 & C(\theta, 0) \text { is finite } \\
\text { at } \theta=0 & C(0, r)=0 \\
& \\
& C_{g}(0)=C_{g I}
\end{array}
$$

Using the Laplace transformation method, the solutions of Eqs. (8) and (10) under the conditions of Eq. (12) are obtained as follows.

$$
\begin{aligned}
\frac{\bar{C}(\theta)}{C_{g I}} & =\frac{1}{(4 / 3) \pi r_{p}^{3}} \int_{0}^{r_{p}} 4 \pi r^{2} C(\theta, r) d r \\
& =\frac{1}{1+f_{1}}-6 f_{2}^{2} \sum_{n=1}^{\infty} \frac{\exp \left(-f_{3} \beta_{n}^{2} \theta\right)}{F\left(\beta_{n}\right)} \\
\frac{C_{g}(\theta)}{C_{g I}} & =\frac{1}{1+f_{1}}+18 f_{1} f_{2}^{2} \sum_{n=1}^{\infty} \frac{\exp \left(-f_{3} \beta_{n}^{2} \theta\right)}{F\left(\beta_{n}\right)}
\end{aligned}
$$

where

$$
\begin{aligned}
& f_{1}=m(W / G), \quad f_{2}=k_{g} r_{p} / \mathscr{D}_{e}, \quad f_{3}=\mathscr{D}_{e} /\left(m r_{p}^{2}\right) \\
& F\left(\beta_{n}\right)=\beta_{n}^{4}-\left\{6 f_{1} f_{2}-f_{2}\left(f_{2}-1\right)\right\} \beta_{n}^{2}+9 f_{1} f_{2}^{2}\left(1+f_{1}\right)
\end{aligned}
$$

and $\beta_{n}$ is the $n$-th positive root of

$$
\frac{\tan y}{y}=\frac{3 f_{1} f_{2}-y^{2}}{3 f_{1} f_{2}+\left(f_{2}-1\right) y^{2}}
$$

If $\bar{C}(\theta) / C_{g 1}$ and $C_{g}(\theta) / C_{g I}$ are replaced by $\bar{t}(\theta) / t_{g I}$ and $t_{g}(\theta) / t_{g I}$, respectively, Eqs. (13) and (14) are the solutions for the case of heat transfer, where

$f_{1}=\left(C_{p} \rho_{p} / C_{f} \rho_{f}\right)(W / G), \quad f_{2}=h r_{p} / k_{p}, \quad f_{3}=k_{p} /\left(C_{p} \rho_{p} r_{p}^{2}\right)$

and the value of $h$ is calculated by the equation ${ }^{10)}$

$$
N u=2+0.60 \operatorname{Re}^{1 / 2} \operatorname{Pr}^{1 / 3}
$$

The residence time of particle $\theta_{p}$ or gas $\theta_{f}$ in the cloud is calculated as follows.

$$
\theta_{p}=\pi\left(R+D_{b} / 2\right) /\left(2 u_{p}\right), \quad \theta_{f}=\theta_{p}\left\{\varepsilon_{m f} /\left(1-\varepsilon_{m f}\right)\right\}(W / G)
$$

$W$ and $G$ are estimated by Davidson's theory ${ }^{1)}$, for a two-dimensional bubble

$$
\begin{aligned}
& W=2\left(1-\varepsilon_{m f}\right) u_{b}\left\{1-\left(D_{b} / 2 R\right)^{2}\right\} R \\
& G=2 u_{m f} D_{b}
\end{aligned}
$$

and for a three-dimensional bubble

$$
\begin{aligned}
& W=\left(1-\varepsilon_{m f}\right) u_{b}\left\{1-\left(D_{b} / 2 R\right)^{3}\right\} R^{2} / 2 \\
& G=3 u_{m f}\left(\pi D_{b}^{2} / 4\right)
\end{aligned}
$$


Table 1 Physical properties of activated alumina

\begin{tabular}{|c|c|c|c|}
\hline Particles & $-48+65^{*}$ & $-65+80$ & $-80+100$ \\
\hline$r_{p}[\mu]$ & 126 & 96 & 80.5 \\
\hline$u_{m f}[\mathrm{~cm} / \mathrm{sec}]$ & 6.1 & 4.4 & 2.7 \\
\hline$\varepsilon_{m f}[-]$ & 0.400 & 0.391 & 0.371 \\
\hline$m$ & 96.7 & 79.4 & 81.5 \\
\hline Gas & \multicolumn{3}{|c|}{$\mathrm{N}_{2}-$ Freon 12 system } \\
\hline $\mathscr{D} e\left[\mathrm{~cm}^{2} / \mathrm{sec}\right]$ & \multirow{3}{*}{\multicolumn{3}{|c|}{$\begin{array}{l}7.5 \times 10^{-5} \\
9.0 \times 10^{-2}\end{array}$}} \\
\hline $\mathscr{D}_{g}\left[\mathrm{~cm}^{2} / \mathrm{sec}\right]$ & & & \\
\hline *) Tyler mesh & & & \\
\hline
\end{tabular}

Table 2 Physical properties of glass beads

\begin{tabular}{lccc} 
Particles & $-48+60 *)$ & $-60+80$ & $-80+100$ \\
\hline$r_{p}[\mu]$ & 135 & 108 & 80.5 \\
$u_{m f}[\mathrm{~cm} / \mathrm{sec}]$ & 5.77 & 3.00 & 1.96 \\
\hline$\varepsilon_{m f}[-]$ & & 0.40 & \\
$k_{p}\left[\mathrm{cal} / \mathrm{cm} \cdot \mathrm{sec}^{\circ} \mathrm{C}\right]$ & & 2.22 & \\
$C_{p}\left[\mathrm{cal} / \mathrm{g} \cdot{ }^{\circ} \mathrm{C}\right]$ & & 0.184 & \\
$\rho_{p}[\mathrm{~g} / \mathrm{cc}]$ & 2.17 & \\
${ }^{*} \quad$ Tyler mesh & & \\
\hline
\end{tabular}

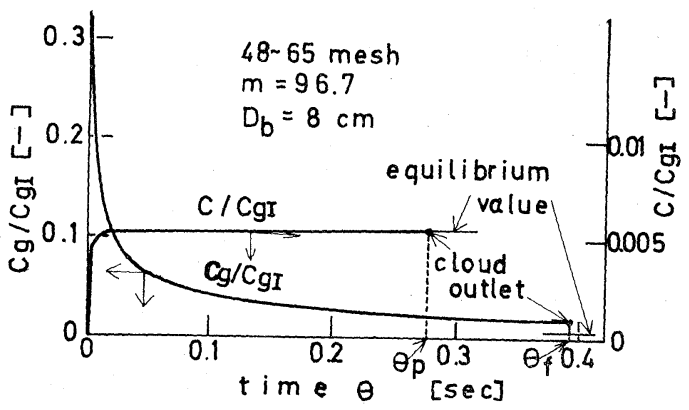

Fig. 3 The change of $C_{g}$ and $\bar{C}$ with $\theta$ in cloud

In Eqs.(21), (23), the values of $W$ are calculated at $90^{\circ}$ from the top of the cloud, and are maximum values. Other methods of estimating. $W$ are also available, such as taking the average value of $W$ in the cloud. But it does not much affect the final result.

The change of $\bar{C}(\theta)$ and $C_{g}(\theta)$ with contacting time $\theta$ for the case of a two-dimensional bubble are calculated under the conditions tabulated in Table 1 (48 65 mesh), and the results are shown in Fig. 3. The concentration of adsorptive gas in the circulating gas decreases quite rapidly, although it takes a long time to approach the equilibrium value, and at the outlet of the cloud it can be said that nearly the whole amount of adsorptive gas is adsorbed by particles and the gas concentration becomes nearly zero. From this, it can be said that the third assumption is correct.

The change of temperatures $\bar{t}(\theta)$ and $t_{g}(\theta)$ are also calculated for the case of a three-dimensional bubble under the conditions tabulated in Table 2 (glass beads $48 \sim 60$ mesh). The temperature of the circulating gas through the cloud very rapidly approaches zero (at $\theta=10^{-3} \mathrm{sec}, t_{g} / t_{9 I}=8 \times 10^{-3}$ ) and at the cloud outlet the temperature becomes the equilibrium temperature $\left(t_{g} / t_{g I}=1.34 \times 10^{-3}\right)$.

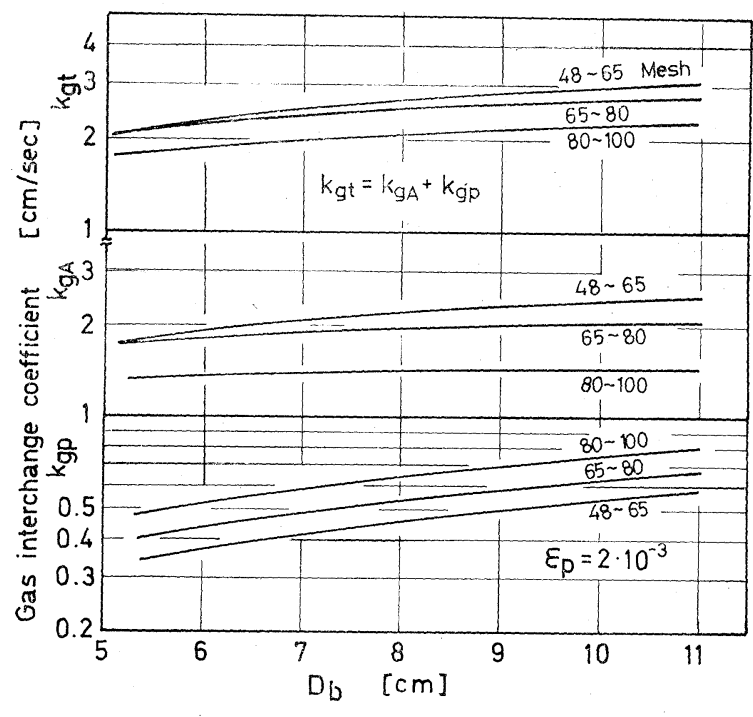

Fig. 4 Calculated gas interchange coefficients $\left(\boldsymbol{k}_{g A}, \boldsymbol{k}_{g p}\right.$ \& $k_{g t}$ )(physical properties of particles and gas are given in Table 1)

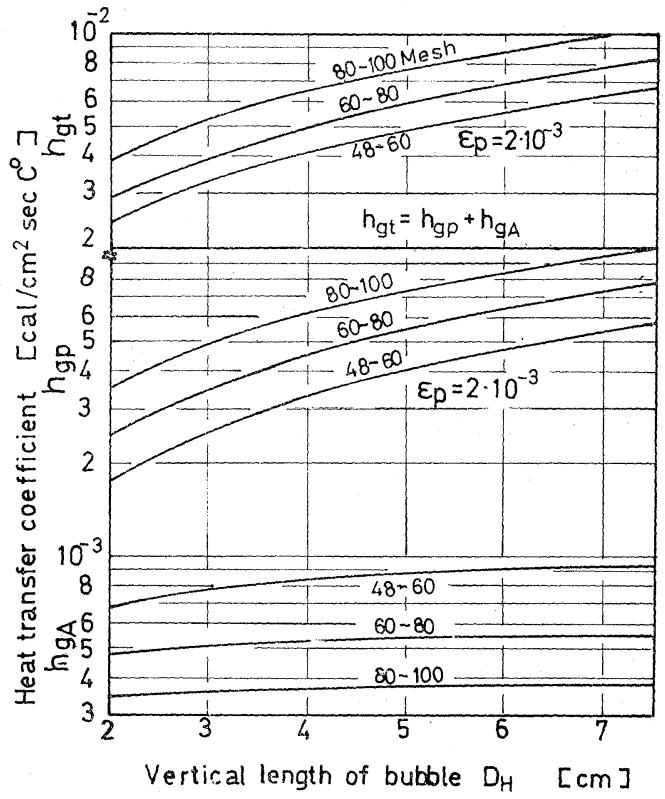

Fig. 5 Calculated heat transfer coefficient $\left(h_{g p}, h_{g A} \&\right.$ $h_{g t}$ ) (physical properties of particles are given in Table 2)

The gas adsorption rate $N_{A}$ and heat transfer rate $Q_{A}$, respectively, to the particles in the cloud are given as follows.

$$
\begin{aligned}
N_{A} & =G\left(C_{g I}-C_{g o u t}\right) \doteqdot G C_{g I} \\
& =2 u_{m f} D_{b} C_{g I} \\
Q_{A} & =C_{f} \rho_{f} G\left(t_{g I}-t_{\text {gout }}\right) \doteqdot C_{f} \rho_{f} G t_{g I} \\
& \doteqdot(3 / 4) \pi D_{b}^{2} C_{f} \rho_{f} u_{m f} t_{g I}
\end{aligned}
$$

From Eqs. (25) and (26), it can be said that the transfer rates are proportional to the circulation rate of gas in bubble $G$. In other words, they are restricted by the circulation rate $G$.

The gas interchange coefficient $k_{g A}$ for a two-dimensional bubble and heat transfer coefficient $h_{g A}$ for a 


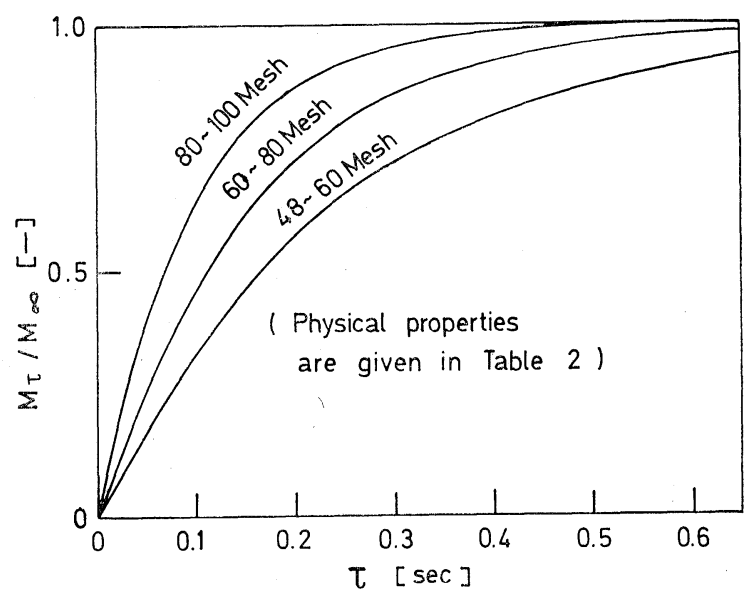

Fig. 6 The degree of approach to equilibrium temperature

three-dimensional bubble per unit surface area of a bubble by this effect, respectively, are

$$
\begin{aligned}
& k_{g A}=\frac{1}{1+2 \varepsilon_{m f} /(\alpha-1)} \frac{2 u_{m f}}{\pi} \\
& h_{g A}=\frac{1}{1+3 \varepsilon_{m f} /(\alpha-1)} \frac{3}{4} C_{f} \rho_{f} u_{m f}
\end{aligned}
$$

The calculated values of $k_{g A}$ by Eq.(27) and $h_{g A}$ by Eq.(28) to the bubble diameter for various particle diameters are shown in Figs. 4. and 5, respectively. The values of $k_{g_{A}}$ and $h_{g_{A}}$ increase as the bubble diameter or the particle diameter increases because of increasing $\alpha$ or $u_{m f}$, respectively.

1.2 The diffusion or heat conduction from the cloud surface and the periodical shedding-out of gas from the cloud

As shown in above calculations and in Fig. 3, the concentration or the temperature of the circulating gas in the cloud becomes very low near the top of the bubble. So the gas interchange coefficients $k_{g_{D}}$ and $k_{g C}$ or the heat transfer coefficients $h_{g D}$ and $h_{g C}$ by these effects can be neglected.

\subsection{The capacitance effect of particles falling in a bubble}

The volumetric fraction $\varepsilon_{p}$ of particles in a bubble was investigated by many workers ${ }^{4,5,13)}$, and it was concluded that the value of $\varepsilon_{p}$ is as small as the order of $10^{-3}$. But the heat capacity ratio of particle to gas $C_{p} \rho_{p} / C_{f} \rho_{f}$ is on the order of $10^{3}$. So the total heat capacity of particles in a bubble is on the same order as that of gas in a bubble. Therefore the role of particles in a bubble becomes important.

The amount of heat absorbed by particles in a bubble per unit time $Q_{p}$ is approximated by

$$
Q_{p}=u_{b} A^{\prime} \varepsilon_{p} C_{p} \rho_{p}\left(M_{\tau} / M_{\infty}\right) t_{g}
$$

where $A^{\prime}$ is the cross sectional area of bubble, for the two-dimensional bubble

$$
A^{\prime}=D_{b} d
$$

and for the three-dimensional bubble

$$
A^{\prime}=(\pi / 4) D_{b}^{2}
$$

and $M_{\tau} / M_{\infty}$ is the degree of approach to the heat equilibrium when temperature of gas in a bubble is uniform, and the value of $M_{\tau} / M_{\infty}$ can be calculated by solving the partial differential equation considering the conduction in a particle and the convection around the particle.

$$
\frac{M_{r}}{M_{\infty}}=1-6 \sum_{n=1}^{\infty} \frac{\exp \left(-f_{3} \delta_{n}^{2} \tau\right)}{\delta_{n}^{2}\left[\left(\delta_{n} / f_{2}\right)^{2}+\left(1-1 / f_{2}\right)\right]}
$$

where $f_{2}$ and $f_{3}$ are defined by Eq. (18) for heat transfer, and $\delta_{n}$ is the $n$-th positive root of

$$
y \cot y=1-f_{2}
$$

In the case of heat transfer using glass beads, the value of $f_{2}$ is very small (about $4 \times 10^{-2}$ ), therefore the resistance to conduction in a particle can be neglected, so Eq.(32) can be simplified as follows.

$$
M_{\tau} / M_{\infty}=1-\exp \left(-3 f_{2} f_{3} \tau\right)
$$

$\tau$ is the falling time of a particle in a bubble and is approximated to be the time of free fall of a particle under gravity through a bubble. The falling distance through the bubble is taken as $D_{b}$ for a two-dimensional bubble since the bubble is nearly circular, and as $D_{H}$ for a three-dimensional bubble. Then $\tau$ are

$$
\begin{array}{ll}
\tau=\sqrt{2 D_{b} / g} & \text { for two-dimensional bubble } \\
\tau=\sqrt{2 D_{H} / g} & \text { for three-dimensional bubble }
\end{array}
$$

The values of $M_{\tau} / M_{\infty}$ calculated from Eq.(34) are shown in Fig. 6. The degree of approach to equilibrium becomes larger as the particle diameter becomes smaller.

The heat transfer coefficient $h_{g p}$ by this effect is expressed as follows for a three-dimensional bubble.

$$
h_{g p}=(1 / 4) u_{b} \varepsilon_{p} C_{p} \rho_{p}\left(M_{\tau} / M_{\infty}\right)
$$

For the transfer of adsorptive gas, $Q_{p}, C_{p} \rho_{p}$ and $t_{g}$ in Eq.(29) are replaced by $N_{p}, m$, and $C_{g}$, respectively, and $M_{\tau} / M_{\infty}$ is calculated by Eqs.(32) and (15). Finally, the gas interchange coefficient $k_{g p}$ by this effect for a two-dimensional bubble is

$$
k_{g p}=(1 / \pi) m \varepsilon_{p}\left(M_{\tau} / M_{\infty}\right)
$$

The value of $h_{g p}$ from Eq.(37) or $k_{g p}$ from Eq.(38) are shown as a function of the vertical length or the diameter of bubble for various particle diameters in Fig. 5 or 4 . The value of $h_{g p}$ or $k_{g p}$ increases with increasing bubble diameter because of increasing $u_{b}$ and $\tau$ and also increases with decreasing particle diameter because of increasing value of $M_{\tau} / M_{\infty}$. In the calculaticns of Eqs.(37) and (38), the volumetric fraction of particles $\varepsilon_{p}$ is taken as $2 \times 10^{-3}$, obtained in the authors' previous work ${ }^{13)}$ for a two-dimensional bubble.

\subsection{The total coefficients}

Assuming the effects mentioned above act independently and in parallel, the total coefficient of gas interchange $k_{g t}$ or heat transfer $h_{g t}$ between a bubble and the continuous phase is expressed as follows.

$$
\begin{aligned}
& k_{g t}=k_{g_{A}}+k_{g D}+k_{g C}+k_{g p} \doteqdot k_{g A}+k_{g p} \\
& h_{g t}=h_{g A}+h_{g D}+h_{g C}+h_{g p} \doteqdot h_{g A}+h_{g p}
\end{aligned}
$$

The values of $k_{g t}$ calculated by Eq.(39) are shown in Fig. 4. The contribution of $k_{g A}$ to $k_{g t}$ is considerably larger than that of $k_{g p}$. Particularly, in the case of coarse particles, $k_{g A}$ almost controls $k_{g t}$. The calcu- 


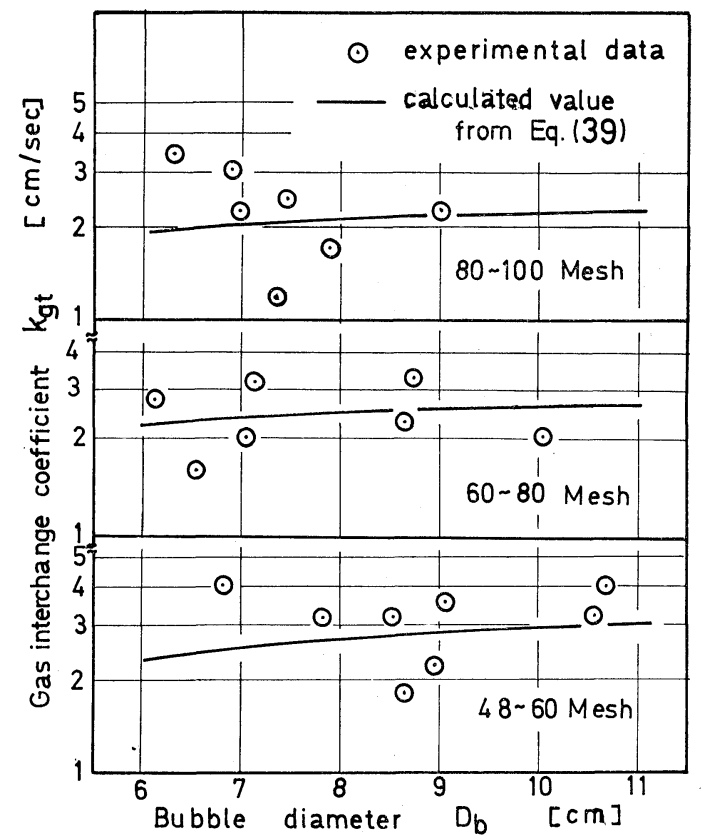

Fig. 7 Experimental and calculated values of $k_{g t}$ (activated alumina particles)

lated values of $h_{g t}$ are also shown in Fig. 5. The contribution of $h_{g p}$ to $h_{g t}$ is considerably larger than that of $h_{g A}$ and particularly for fine particles $h_{g p}$ controls $h_{g t}$.

\section{Experimental Procedures and Results}

\subsection{The adsorption experiment}

The same two-dimensional fluidized bed as reported previously $\left.^{12}\right)(49 \mathrm{~cm}$ wide, $130 \mathrm{~cm}$ high, and $2 \mathrm{~cm}$ thick) was used. Activated alumina particles of $48 \sim 100$ mesh were used as fluidized particles. Physical properties of the particles and gas are summarized in Table 1 and the values of the equilibrium constant $m$, measured by B. E. T. equipment, are also tabulated.

The activated alumina particles were carefully packed in the bed so as not to adsorb moisture and the bed was maintained close to the minimum fluidization state by nitrogen gas. Into the bed, a single bubble of Freon 12 (dichlorodifluoromethane) and nitrogen gas mixture (concentration is about 30\%) was injected from a nozzle installed $5 \mathrm{~cm}$ above the distributor of the perforated plate. At two different heights, $40 \mathrm{~cm}$ and $70 \mathrm{~cm}$ from the nozzle, about $2 \mathrm{cc}$ of gas were sucked directly from the rising bubble. The concentration of Freon 12 was analyzed by gas chromatography. The sampling equipment and procedure were the same as reported in authors' previous report ${ }^{12}$. The behaviour of the fluidized bed was recorded by a $16 \mathrm{~mm}$ cine camera (64 frames/sec) under counterlight to evaluate the shape and rising velocity of a bubble and also to confirm that the gas sampling was carried out correctly from the bubble.

Assuming that the shape of the bubble is a circle with diameter $D_{b}$ and relating the ascent distance of bubble $l$ to time by the relation $u_{b} d \theta=d l$, Eq.(1)

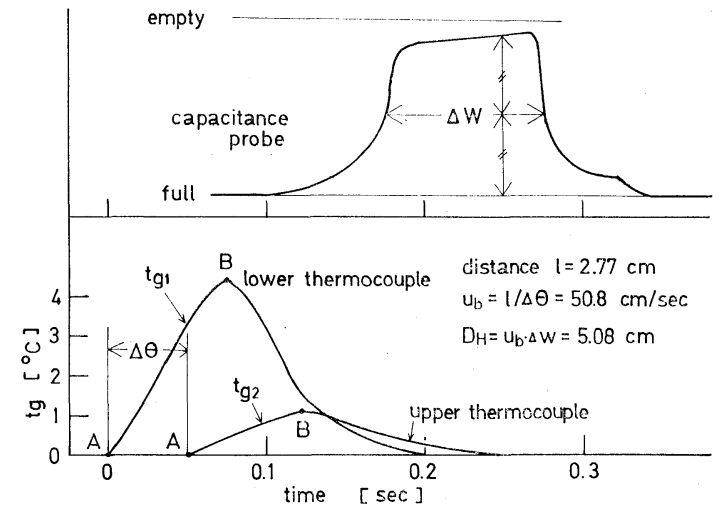

Fig. 8 An example of outputs from thermocouples and capacitance probe

can be integrated as follows.

$$
k_{g t}=\frac{D_{b} u_{b}}{4 l} \ln \left(\frac{C_{g_{1}}}{C_{g_{2}}}\right)
$$

where $C_{g 1}$ and $C_{g 2}$ are the Freon 12 gas concentrations at the lower and higher sampling points, respectively.

From Eq.(41), the gas interchange coefficient $k_{g t}$ was evaluated per unit surface area of a bubble and are shown in Fig. 7 with the theoretical $k_{g t}$ from Eq. (39). The data scatters considerably, but the agreement between experimental and theoretical values is fairly good. It may, therefore, be concluded that the model is appropriate.

\subsection{The heat transfer experiment}

A three-dimensional fluidized bed $(10 \times 10 \mathrm{~cm}$, $100 \mathrm{~cm}$ high) made of transparent P.V.C. was used. The calming section was made of a packed bed $(7 \mathrm{~cm}$ high) of sand. Glass beads were used as fluidized particles, and their physical properties are shown in Table 2. The bed was maintained close to the minimum fluidization state by air and a single bubble of high-temperature gas was injected from a nozzle $14 \mathrm{~cm}$ above the distributor (the perforated plate). At two different heights, about $7 \mathrm{~cm}$ and $10 \mathrm{~cm}$ from the nozzle, the temperature of the gas in the bubble was measured by copper-constantan thermocouples $($ diameter $=40 \mu)$. The distance $l$ between two thermocouples was $2.77 \mathrm{~cm}$. The bubble diameter was measured at $16 \mathrm{~cm}$ above the nozzle by capacitance method. The output signals from thermocouples and capacitance probe were amplified and recorded by a pen-writing oscillograph. An example of output signals is shown in Fig. 8. From observations in a two-dimensional bed, it was confirmed that the thermocouple entered into a bubble at point $\mathrm{A}$ and at point $B$ it was near the centre of the bubble. Therefore the temperature at point $\mathrm{B}$ was taken as that of the bubble. But two points should be noted. The first is that the time lag of the thermocouple is considerable, as seen in Fig. 8, and it is a question whether point $\mathrm{B}$ in Fig. 8 shows the true temperature or not. This point can be overcome as follows. The temperature of the gas in the bubble decreases as the bubble rises by losing the heat to the continuous phase, 


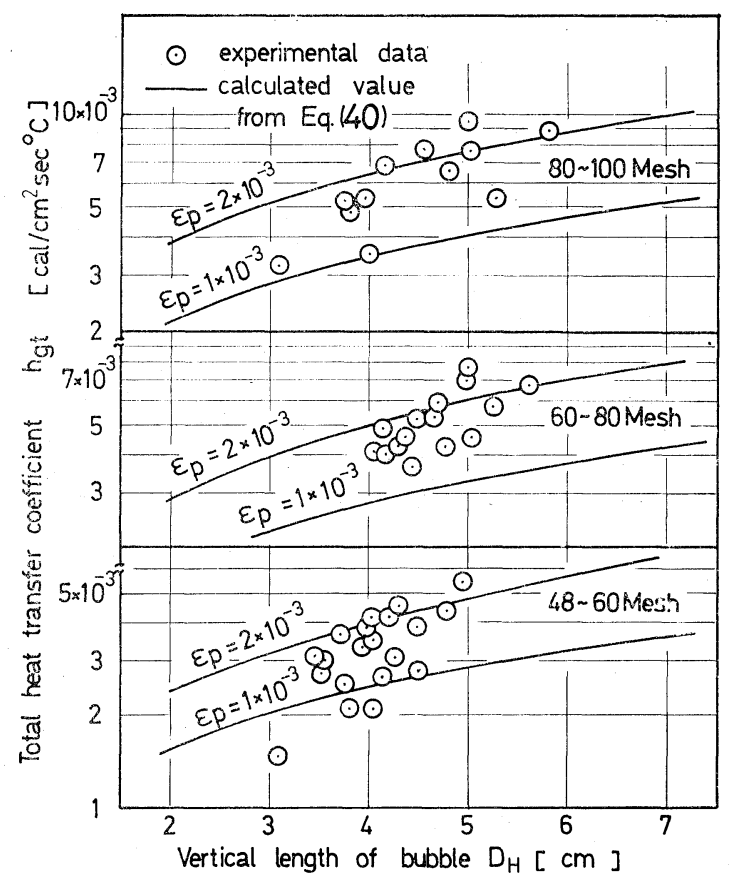

Fig. 9 Experimental and calculated values of $h_{g t}$ (glass beads)

while the temperature of the thermocouple increases by getting heat from the gas. Since the thermocouple has a time lag, these temperatures cross at a certain time. Just after the crossing, the temperature of the latter will decrease since the driving force of the heat transfer from gas will reverse. Therefore the crossing point is the maximum temperature that the thermocouple attains, and it shows the true temperature of the gas. This crossing point should be B in Fig. 8 . The second point is whether the assumption of uniform temperature in the bubble is appropriate or not, since the decreasing rate of gas temperature in the bubble is so fast as seen in Fig. 8. At the present stage, there is no resolution of this point. From Fig. 8 , the vertical length $D_{H}$ and the velocity $u_{b}$ of the bubble was calculated as follows.

$$
u_{b}=l / \Delta \theta, \quad D_{H}=u_{b} \cdot \Delta w
$$

The total heat transfer coefficient $h_{g t}$ is obtained by integrating Eq.(2) as follows.

$$
h_{g t}=\frac{D_{H} u_{b} C_{f} \rho_{f}}{4.83} \ln \left(\frac{t_{g 1}}{t_{g 2}}\right)
$$

where $t_{g 1}$ and $t_{g 2}$ are the temperatures of the bubble at the lower and higher measuring points, respectively. From Eq. (43), the heat transfer coefficient $h_{g t}$ per unit surface area of the sphere of diameter $D_{b}$ was evaluated and is shown in Fig. 9 with the theoretical value from Eq. (40) shown by solid lines with parameter $\varepsilon_{p}=1$ $\times 10^{-3}$ and $2 \times 10^{-3}$. The data scatter considerably but are in the range of $\varepsilon_{p}=(1 \sim 2) \times 10^{-3}$ of the theoretical results. The agreement between experimental and theoretical values is quite good.

\section{Conclusion}

It can be concluded from the authors' theoretical model that there are two kinds of contributions of particle flow to transfer mechanism, classified by the amount of the adsorptive or heat capacity of the particles.

1) When the adsorptive equilibrium constant $m$ or the heat capacity ratio of particles to gas $C_{p} \rho_{p} /$ $C_{f} \rho_{f}$ is of the order of $10 \sim 10^{2}$, the effect of particles flowing through the cloud controls the transfer rate. Consequently, the circulating rate of gas between cloud and bubble $G$ controls the transfer rate. In this paper, $G$ is taken as that predicted by the Davidson's theory' ${ }^{1)}$.

2) When $m$ or $C_{p} \rho_{p} / C_{f} \rho_{f}$ is of the order of $10^{3}$, the effect of particles falling in the bubble controls the transfer rate, and the volumetric fraction of particles $\varepsilon_{p}$ in the bubble is important. In this paper, $\varepsilon_{p}$ is taken as $(1 \sim 2) \times 10^{-3}$.

An experiment on the transfer of adsorptive gas between a two-dimensional bubble and the continuous phase was performed and the agreement between theoretical model and experimental results was fairly good. In consequence, it was found that their model is appropriate for case $^{1)}$. The theoretical model for case 2) was checked by an experiment on the heat transfer between a three-dimensional bubble and the continuous phase. Again the agreement was fairly good, so their model for this case and also the value of $\varepsilon_{y}=(1 \sim 2) \times 10^{-3}$ seems appropriate.

\section{Nomenclature}

$A \quad=$ width of cloud $[\mathrm{cm}]$

$A_{b} \quad=$ surface area of bubble $\left[\mathrm{cm}^{2}\right]$

$A^{\prime} \quad=$ cross-sectional area of bubble $\quad\left[\mathrm{cm}^{2}\right]$

$C=$ concentration of adsorptive gas in particle in

$\bar{C} \quad$ cloud [mole/cc]

$\bar{C} \quad=$ average concentration of adsorptive gas in

particle in cloud [mole/cc]

$C_{e}=$ concentration of adsorptive gas in continuous

phase [mole/cc]

$C_{g} \quad=$ concentration of adsorptive gas in circulating gas

through cloud or in bubble [mole/cc]

$C_{s}=$ concentration of adsorptive gas adsorbed by particle [mole/cc]

$C_{f}, C_{p}=$ specific heat of gas or particle $\quad\left[\mathrm{cal} / \mathrm{g} \cdot{ }^{\circ} \mathrm{C}\right]$

$D_{b}=$ diameter of bubble $\quad[\mathrm{cm}]$

$d \quad=$ thickenss of the two-dimensional bed $\quad[\mathrm{cm}]$

$\mathscr{D}_{e} \quad=$ effective diffusivity in particle $\quad\left[\mathrm{cm}^{2} / \mathrm{sec}\right]$

$\mathscr{D}_{g}=$ diffusivity in $\mathrm{N}_{2}$-Freon $12 \quad\left[\mathrm{~cm}^{2} / \mathrm{sec}\right]$

$D_{H} \quad=$ vertical length of bubble
$[\mathrm{cm}]$

$G \quad=$ flow rate of circulating gas through cloud $g \quad=$ gravitational acceleration $(=980)$

$k_{g} \quad=$ mass transfer coefficient around particle $\left[\mathrm{cm} / \mathrm{sec}^{2}\right]$

$k_{g_{t}}, k_{g_{A}}, h_{g_{D}}, k_{g C}, k_{g p}=$ gas interchange coefficient between bubble and continuous phase

$k_{p} \quad=$ thermal conductivity of particle

$[\mathrm{cm} / \mathrm{sec}]$ $=$ heat transfer coefficient around particle

$\left[\mathrm{cal} / \mathrm{cm}^{2} \cdot \mathrm{sec} \cdot{ }^{\circ} \mathrm{C}\right]$ 


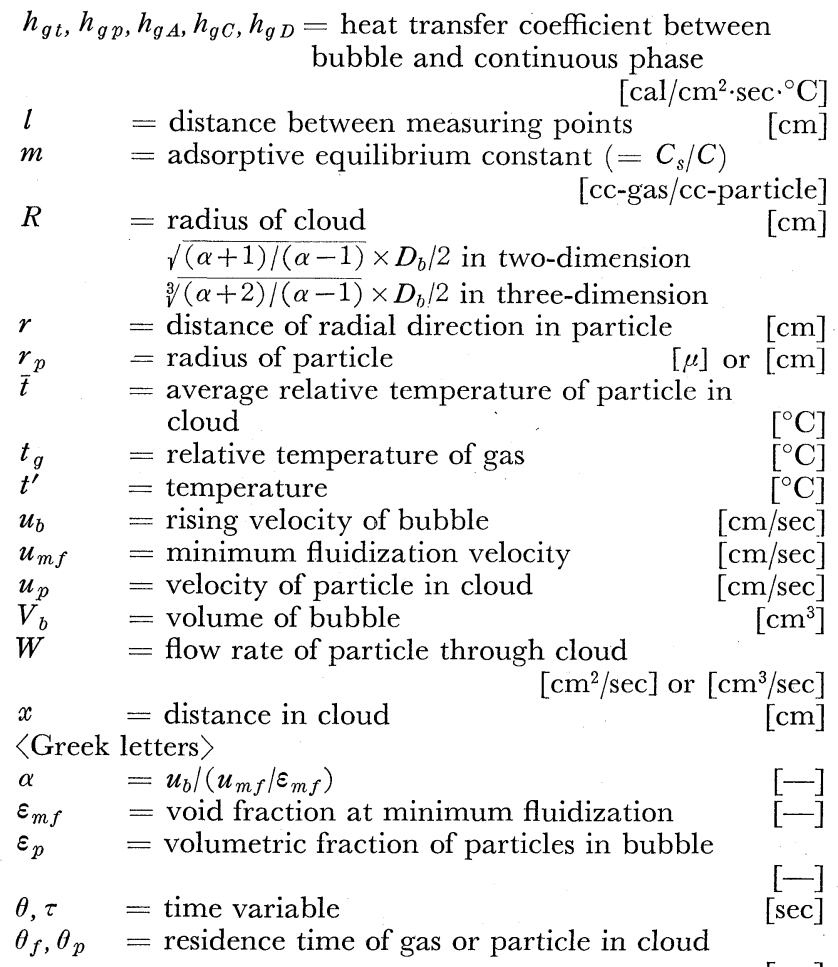

[sec] $\rho_{f}, \rho_{p}=$ density of gas or particle

$\left[\mathrm{g} / \mathrm{cm}^{3}\right]$

\section{Literature Cited}

1) Davidson, J. F. and D. Harrison: "Fluidised Particles", Cambridge University Press (1963)

2) Gilliland, E. R. and E. A. Mason: Ind. Eng. Chem., 44, $218(1952)$

3) Kobayashi, H., F. Arai and T. Sunagawa: Kagaku Kōgaku, 31, 239 (1967)

4) Kobayashi, H., F. Arai and T. Chiba: ibid., 29, 858 (1965)

5) Kunii, D., K. Yoshida and I. Hiraki: ibid., 29, 846 (1965)

6) Kunii, D. and T. Wakabayashi: J. Chem. Eng. Japan, 4, 226 (1971)

7) Miyauchi, T.: Prepringt of the 30th Annual Meeting of the Soc. of Chem. Engrs., Japan, 447 (1965)

8) Miyauchi, T. and S. Morooka: Kagaku Kōgaku, 33, 88 (1969)

9) Overscashier, R. H., D. B. Tood and R. B. Olney: A.I.Ch.E. Journal, 5, 54 (1959)

10) Ranz, W. E. and W. R. Marshall, Jr.: Chem. Eng. Prog., 48, 141, 173 (1952)

11) Richardson, J. F. and L. Davies: Trans. Instn. Chem. Engrs., 44, 293 (1966)

12) Toei, R., R. Matsuno, H. Miyagawa, K. Nishitani and Y. Komagawa: Kagaku Kōgaku, 32, 565 (1968)

13) Toei, R., R. Matsuno, H. Kojima, K. Nakagawa and S. Yu: ibid., 29, 851 (1965)

\title{
A NEW DEVICE FOR PNEUMATIC TRANSPORT OF PARTICLES
}

\author{
KUNIO SHINOHARA AND TATSUO TANAKA \\ Department of Chemical Process Engineering, Hokkaido \\ University, Sapporo, Japan
}

\begin{abstract}
A new device has been developed to convey particles through a duct with air or gas.
The pneumatic apparatus has an air box under a porous plate on which many inclined parallel plates are attached to orientate and convey particles from one cell to the next cell with air or gas. Thus, particles can be transported upwards as well as downwards. The average residence time or the apparent passing velocity of particles, the pressure drop of air or gas, and the power required to convey solids are investigated by applying the method of dimensional analysis. A minimum transport velocity of air is also evaluated, assuming a minimum voidage of particles mass within cells.

As a result, they are expressed as functions of geometrical dimensions of the device, physical properties of particles, and of operational conditions such as the feed rate of particles, the inclination angle of the device and flow rate of air or gas. Experimental results verify the derived equations.
\end{abstract}

\section{Introduction}

Roughly speaking, there are three types of transport of solids at present, namely mechanical transport, fluid transport and vessel transport. Among them, the so-called pneumatic conveyor and Air-slide are

* Received on December 24, 1971

Presented at the 34th Annual Meeting of the Soc. of Chem. Engrs., Japan, April 4, 1969 widely used as a type of fluid transport, especially in the chemical industry for handling dry bulk solids. The merits of these pneumatic devices are large capacity and long distance of transport, easy conveyance even through a complex channel with small space, no dispersion of powdery dust, and so on. But the demerit of the so-called pneumatic conveyor is the large power consumption, while the essential demerit of Air-slide is the fact that it is incapable of convey- 